\title{
Desenvolvimento de lista de boas práticas para o processo de elevação da alvenaria
}

\author{
Development of list of best practices for the process of masonry elevation \\ V. F. de Mesquita ${ }^{1}$; D. de G. Santos ${ }^{2 *}$ \\ ${ }^{1}$ Coordenadoria de Obras e Projetos, Universidade Federal do Ceará, 60020-181, Fortaleza - CEARÁ, Brasil \\ ${ }^{2}$ Departamento de Engenharia Civil, Universidade Federal de Sergipe, 49.100-000, São Cristóvão - SERGIPE, Brasil \\ *deboragois@yahoo.com.br \\ (Recebido em 10 de junho de 2015; aceito em 29 de outubro de 2015)
}

\begin{abstract}
As empresas construtoras necessitam aplicar conceitos, métodos e técnicas enxutas, para a identificação de atividades que não agregam valor ao produto. Nesse contexto, inserem-se as atividades que contribuem para a melhoria dos processos construtivos. São as boas práticas que buscam eliminar fatores que gerem interrupções nos processos que ocorrem nos canteiros de obras. $\mathrm{O}$ objetivo deste estudo consiste em desenvolver uma lista de boas práticas para o processo de elevação da alvenaria estrutural, verificando a frequência de aplicação dessas atividades nos canteiros de obras. O método de trabalho adotado foi o de realização de entrevistas não estruturadas com os gerentes de obras, visitas técnicas e aplicação de lista de verificação para investigar a importância que os gestores dão ao uso de atividades que facilitem o trabalho. Como resultado, foi elaborada uma lista de boas práticas. Ao analisar a lista foi constatado que a negligência da maioria dessas atividades poderia resultar na ocorrência de perdas por making-do e, consequentemente, em retrabalhos. A aplicação do checklist nos canteiros mostrou que, mesmo sem perceber, os gerentes utilizam as boas práticas no seu dia a dia.
\end{abstract}

Palavras-chave: boas práticas, atividades facilitadoras, construção enxuta.

Construction companies seek improvements for their management systems. In this context, there are activities that contribute to the improvement of construction processes. They are best practices that seek to eliminate factors that create interruptions in processes that occur at construction sites. The aim of this study is to develop a list of best practices for the masonry process by checking the frequency of implementation of these activities at construction sites. The adopted method consisted in unstructured interviews with the works managers, technical visits and checklist application to investigate the importance of the application of activities that work facilitation. As a result, a list of best practices was drafted. By analyzing this list was found that the neglect of most of these activities could result in the occurrence of making-do and consequently in rework. The application of checklist showed that the managers use best practices in their daily.

Keywords: Best practices; Extra-planning activities; Lean construction; Games and simulations.

\section{INTRODUÇÃO}

As empresas construtoras buscam melhorias de seus sistemas gerenciais. Para tanto, necessitam aplicar conceitos, métodos e técnicas enxutas, para a identificação e posterior eliminação ou redução de atividades que não agregam valor ao produto final. Nesse contexto, inserem-se as boas práticas, as quais buscam eliminar fatores que gerem, direta ou indiretamente, interrupções nos processos que ocorrem nos canteiros de obras, evitando a ocorrência de perdas.

Enquanto o saber técnico do operário é passado de um operário para outro com a prática cotidiana, o conhecimento tácito do gerente normalmente não é passado para outros. Isto acontece porque o conhecimento tácito é difícil de ser explicado, sendo inerente às habilidades de uma pessoa. Isto significa que nem gerentes e nem operários, ao aprenderem com boas práticas em seus canteiros de obras, disseminam estas ações para outras pessoas [1].

Assim, as atividades facilitadoras e as antecipações gerenciais, abordadas, respectivamente, por Santos (2004) [2] e Machado (2003) [3], são consideradas como boas práticas e integram esse rol de atividades. O diagrama da Figura 1 [4] apresenta uma contextualização dessas atividades e suas 
principais delimitações. As "boas práticas" englobam exemplos de atividades ou ações que contribuem para a melhoria do trabalho desenvolvido, como atividades facilitadoras, antecipações, antecipações gerenciais, ações gerenciais, entre outras. Já as "atividades facilitadoras" podem ser divididas em dois subconjuntos: "antecipações gerenciais" e "remoção de restrição".

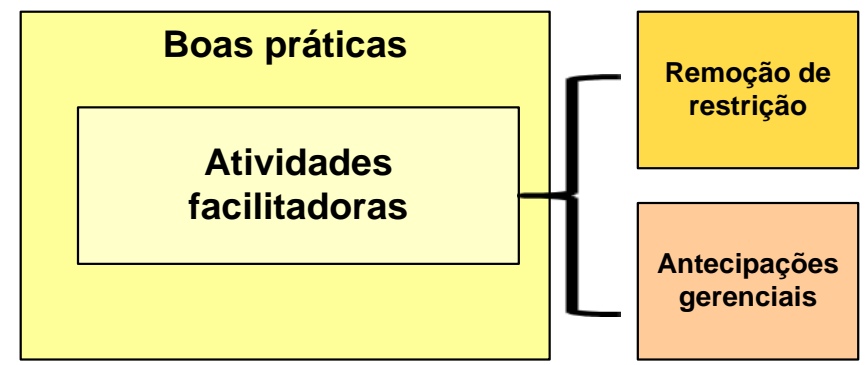

Figura 1: Delimitações das atividades que contribuem para a melhoria dos processos construtivos [4].

Define-se atividade facilitadora como sendo aquelas que quando colocadas no sistema de produção impedem ou minimizam as paradas, ou seja, as interrupções ao longo do processo produtivo [2]. Elas podem estar incorporadas no processo ou auxiliá-lo. Apresentam-se como atividades de produção, de apoio à produção ou ainda como informações [2].

As atividades antecipáveis são definidas como as que representam as ações da gerência que poderiam ser feitas a qualquer momento, para minimizar ou eliminar o impacto causado por interrupções na execução dos processos produtivos [2].

Machado (2003) [3] define antecipações gerenciais como as antecipações que trataram de aspectos mais visíveis, como o provimento de materiais ou de equipamentos, e são de responsabilidade dos gerentes, correlacionando-se com o nível hierárquico do planejamento denominado de nível tático.

Outro termo importante é a restrição. O processo de análise de restrições possibilita o aumento da continuidade das operações no canteiro e a consequente melhoria de eficácia do planejamento, mas os responsáveis pelo processo devem conhecer o desempenho real do sistema e identificar as causas dos principais problemas de obra [5].

Bernardes e Bortolazza (2004, p.13) [6] definem restrição como "todo o tipo de atividade, informação ou elemento que quando não disponível no momento oportuno e na quantidade adequada causa algum tipo de interrupção no fluxo de trabalho no canteiro de obras". Codinhoto et al. (2003) [7] definem restrições como atividades gerenciais, necessidades físicas, financeiras e de informações de projeto, que se não disponibilizadas no momento, na quantidade e especificações corretas, impedem a definição correta dos pacotes de trabalho que the são relacionados. Necessitam, portanto, de um responsável e de uma data limite para removê-las, bem como de uma tarefa a ser executada e atribuída a estas.

Assim, na construção civil, é possível investigar o processo construtivo para contribuir com ações que facilitem o trabalho (work facilitation) [8], por meio da continuidade do fluxo de produção, minimizando a ocorrência de perdas. Estas perdas podem causar restrições ou dificultar o desempenho do trabalho. Liker e Meier (2008) [9] destacam a importância dos pontos chave para a realização do trabalho, contribuindo para evitar a interrupção do trabalho com um treinamento adequado, o que pode levar a ganhos de produtividade.

Ohno (1997) [10] ressalta a importância de identificar e eliminar as perdas no sistema produtivo, separando o trabalho que adiciona valor do que não adiciona valor ao produto. Assim, Shingo (1996) [11] propôs sete categorias de perdas, as quais podem ser aplicadas a qualquer tipo de processo. São perdas por: superprodução; transporte; no processamento em si; estoque; fabricação de produtos defeituosos; movimentação; e espera.

Além dessas categorias de perdas, Koskela (2004) [12] definiu o making-do como o oitavo tipo de perda. O making-do é uma situação na qual a tarefa começa antes que todos os recursos necessários estejam disponíveis para que o trabalho inicie ou continue até seu término.

O making-do foi inspirado na síndrome de eficiência, que é o desejo de utilizar tantos recursos quanto possível [13]. É comum que os gerentes queiram que o pessoal esteja ocupado o tempo 
todo, apenas para que não fiquem ou não aparentem estar ociosos. Isto resulta em mais trabalho em processo, menos rendimento e mais despesas operacionais [13].

A dificuldade em controlar e reunir os recursos a tempo está relacionada com as características intrínsecas aos vários ambientes da produção [12]. Assim, Koskela (2000) [14] afirma que uma tarefa na construção civil é composta por sete pré-requisitos, que são as entradas (inputs) necessárias para começar e continuar uma tarefa. Os sete fluxos são: projetos (informações), materiais/componentes, mão de obra, equipamentos, espaço, tarefas pré-requisito e condições externas.

Sommer (2010) [15] destaca que o termo making-do pode ser definido como a ação de gerenciar com o que se tem disponível, improvisar, ou, em uma linguagem mais coloquial, "dar um jeitinho". Nesse sentido, Fireman (2012) [16] ressalta que o retrabalho merece destaque por apresentar vínculo com algumas das sete categorias, sendo ele identificado como possível consequência da perda por making-do.

Este tipo de perda é frequente nos canteiros de obras e para eliminar ou minimizar essa perda pode-se fazer uso de atividades facilitadoras [2], que se assemelham ao work facilitation.

Baseado no conceito de making-do [12], nos sete fluxos [14] e nas categorias de atividades facilitadoras [2], foram realizados no Brasil uma série de estudos exploratórios a respeito da ocorrência do making-do (Coelho 2009 [17], Sommer 2010 [15], Formoso et al. 2011 [18], Fireman et al. 2013 [19], Fireman 2012 [16], Grosskopf 2013 [20], Mesquita 2014 [4] e Leão 2014 [21]).

Esses estudos forneceram algumas percepções nas limitações dos sistemas de planejamento para evitar o making-do [22]. Os estudos mostraram que essa categoria de perda pode ser considerada como a origem de outros tipos de perda, como problemas de qualidade, trabalho em progresso e retrabalho [22]. Formoso et al. (2011) [18] argumentam que como making-do é consequência de falhas na gestão de processos a montante, é razoável se esperar que existam muitas situações nas quais o funcionário envolvido crie novas formas de realizar a tarefa, utilizando os recursos disponíveis.

Para facilitar o estudo das atividades antecipáveis e de remoção de restrições, as atividades facilitadoras foram divididas em categorias [2], de forma que, ao tentar evitar um erro no desempenho do processo ou detectar uma falha, seja possível a sua classificação. As nove categorias propostas foram:

(1) Acesso - relaciona-se com o acesso de recursos humanos e materiais a locais de trabalho. Compreende o posto de trabalho ou o canteiro de obras. Ele é observado em termos de abastecimentos internos e de alcance da mão de obra à superfície a ser trabalhada;

(2) Projeto - são as características do projeto que possibilitam a sua construtibilidade, como detalhamentos, simplificações, padronizações, compatibilização e conclusões, além de alterações após o início da construção;

(3) Preparação do trabalho - é a disponibilização no posto de trabalho dos recursos de produção, necessários ao início dos processos, em termos de materiais, mão de obra, equipamentos e ferramentas, instruções de trabalho, qualidade da superfície a ser trabalhada e conclusão de processos antecedentes;

(4) Conferência do trabalho - está relacionada com as medidas de desempenho do processo, como tolerâncias dimensionais permitidas, qualidade do produto elaborado e verificação de nivelamentos;

(5) Conflito espacial - relaciona-se com o confronto no espaço de elementos de construção ou de categorias de mão de obra, para a realização de processos diferentes, em um mesmo ambiente de trabalho. Esta categoria pode ser provocada por restrições, que podem ser evitadas com o estudo das movimentações em canteiro e da sequência de execução;

(6) Sequenciamento - está relacionada com a ordem de produção de determinado processo. No caso de uma inversão, observa-se se esta é permitida e quais as providências que devem ser tomadas para evitar a descontinuidade dos processos. Muitas vezes, uma inversão de sequenciamento leva ao aumento no número de visitas ao posto de trabalho, para a conclusão do processo;

(7) Proteção dos operários - trata-se da preocupação com a disponibilização em canteiro de equipamentos de proteção coletivos e individuais. Quanto aos 
primeiros, deve-se verificar a disponibilidade do local adequado para a sua fixação, em termos de tempo e de condições físicas. O trabalho em condição insegura gera risco de acidentes e interrupção das atividades para a devida correção;

(8) Proteção dos processos - observa-se a proteção do serviço de construção já concluído em relação a outros a serem executados no mesmo ambiente e que podem lhe causar danos, como quebras e respingos de materiais;

(9) Programação de obra - esta categoria está relacionada com exigências de clientes fora de hora, desrespeito a planos, definição de pacotes de trabalho, pedido de material, relação com fornecedores e interferência do cliente. Ela ocorre quando uma falha é identificada, mas não é tomada uma providência.

Como as atividades facilitadoras estão incluídas nas boas práticas (Figura 1), pode-se adotar a classificação das atividades segundo as categorias de atividades facilitadoras propostas acima. Estas categorias buscam contribuir para identificar o nível de aplicação das atividades e correlacionar com o tipo de perda a ser minimizado ou eliminado.

Sendo assim, o objetivo deste estudo consiste em desenvolver uma lista de boas práticas para o processo de elevação da alvenaria estrutural, verificando a frequência de aplicação dessas atividades nos canteiros de obras.

\section{MATERIAL E MÉTODOS}

Para a investigação e o levantamento das boas práticas, o presente trabalho adotou como objeto de estudo o processo construtivo de elevação da alvenaria estrutural, por se tratar de atividade considerada gargalo, tanto para o processo de produção como para o sistema de qualidade final das edificações que utilizam a alvenaria portante. A escolha da alvenaria estrutural como objeto de estudo justifica-se também pela abundância destas obras na cidade de Aracaju/Sergipe e na grande Aracaju, o que favoreceu a elevada quantidade de dados pesquisados e a precisão e qualidade das informações coletadas.

O processo de investigação das boas práticas envolveu a realização de entrevistas não estruturadas com engenheiros de seis canteiros de obras; visitas técnicas aos canteiros de obras, o que permitiu visualizar a frequência com que essas atividades eram praticadas no dia a dia dos gestores; e aplicação de uma lista de verificação (checklist), buscando explicitar o conhecimento tácito dos gerentes a respeito da aplicação de boas práticas, segundo as categorias propostas.

O presente estudo utilizou como principais referências para a elaboração da lista de boas práticas: um estudo exploratório realizado durante o período da pesquisa; obras da literatura técnica que tratam de procedimentos executivos de alvenaria estrutural; normas técnicas para a alvenaria estrutural; pesquisas de iniciação científica; artigos científicos; tese de [2] e banco de dados de atividades facilitadoras (Figura 2).

Esta lista foi formatada como um quadro com as seguintes colunas: "item", "frequência (\%) do item", "atividade" (boa prática), "descrição", "causas" (restrições caso a atividade não seja posta em prática), "vantagem" (caso a atividade seja utilizada), "princípio(s) lean relacionado(s)" [22], "categoria(s) de perda" [11, 12], "se não utilizar a atividade o trabalho pode ser interrompido? ou seja, é uma atividade facilitadora?", "categoria de atividade facilitadora" [2], "nível hierárquico do planejamento" e "fonte de pesquisa".

Concluída a lista de boas práticas, procedeu-se à tabulação dos dados através da elaboração de gráficos para permitir a análise estatística dos resultados. 


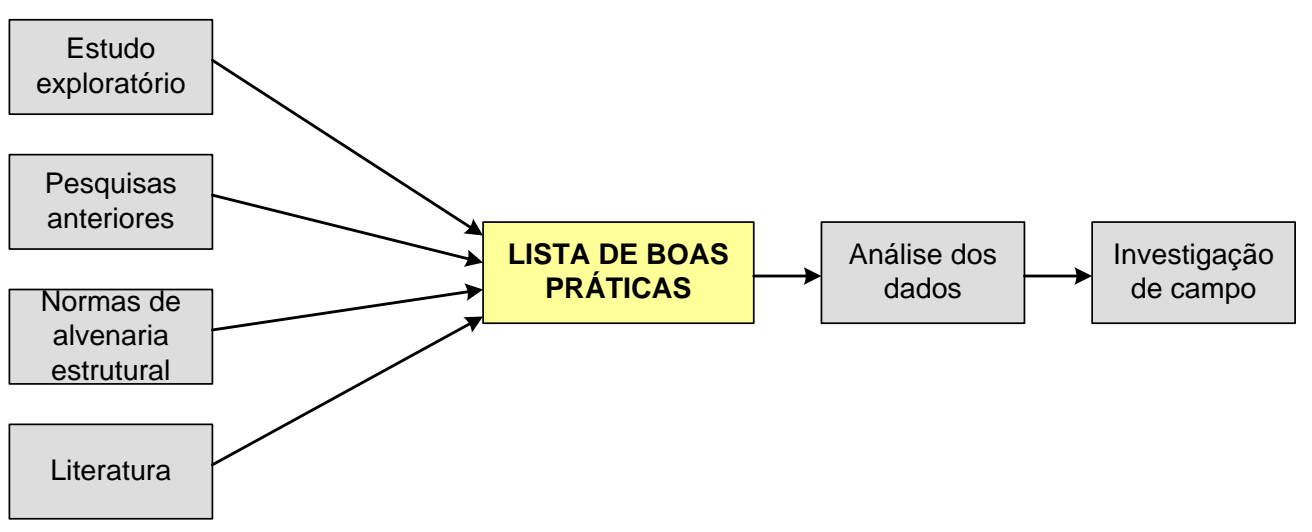

Figura 2 - Diagrama da metodologia da pesquisa

No presente artigo destacam-se os campos: "atividade" (boa prática), "categoria(s) de perda", "é uma atividade facilitadora?", "categoria de atividade facilitadora", "nível hierárquico do planejamento" e "frequência (\%) do item".

Finalmente, realizou-se investigação de campo para verificar a frequência de aplicação das atividades (\% do item) levantadas na lista, através de entrevista não estruturada com gerentes de obras de alvenaria estrutural e observações de campo. Isto significa quantas vezes esse tipo de atividade poderia ter sido aplicado ou foi aplicado nos exemplos identificados.

\section{RESULTADOS E DISCUSSÃO}

Todas as atividades facilitadoras apresentadas na lista foram identificadas como antecipações gerenciais, já que buscam prevenir a ocorrência de situações inesperadas em atividades previsíveis.

As atividades facilitadoras corresponderam a aproximadamente $33 \%$ do total de boas práticas. Esta informação indica que, apesar de existirem dificuldades por parte dos gerentes em aplicar essas atividades, há iniciativa destes em buscar melhorias do processo analisado pela aplicação destas práticas, favoráveis à continuidade do processo.

$\mathrm{Na}$ lista de boas práticas, o nível hierárquico do planejamento para a aplicação das atividades mais frequente foi o operacional, seguindo a lógica de que é no curto prazo onde são visualizados os problemas que deveriam ter sido resolvidos, caso fossem previstos nos níveis de planejamento de médio ou longo prazo.

A investigação de campo mostrou que, mesmo não utilizando algumas boas práticas, no geral, os gestores reconhecem que a sua adoção reduz a ocorrência de perdas e pode trazer benefícios para a continuidade do fluxo.

O Quadro 1 lista exemplos de boas práticas identificadas para o processo construtivo de elevação de alvenaria estrutural, extraídos de uma lista de 63 situações diferentes.

Entre as principais causas de interrupção das atividades, caso as boas práticas levantadas não fossem postas em prática, encontra-se o retrabalho, seguido por falha na programação de tarefas e baixa produtividade. Observou-se, ainda, que estas causas estão relacionadas com as categorias de perda espera e making-do. As demais categorias de perdas identificadas nos exemplos listados foram defeitos, processamento, estoque e transporte. Em termos gerais, sobressaiu-se a perda por making-do, corroborando com o estudo de Fireman (2012) [16].

Com relação às categorias de atividades destacaram-se a de "Preparação do trabalho", "Sequenciamento", "Conferência do trabalho", "Proteção dos operários" e "Acesso". Em termos gerais, ou seja, para os 63 exemplos, destacaram-se as categorias de "Preparação do trabalho", "Projeto" e "Programação de obras".

De modo a confirmar as atividades identificadas na lista de boas práticas e a procurar entender como os gerentes de obras se comportam frente a essas boas práticas, foi aplicada uma lista de verificação (checklist), separando as questões nas categorias de atividades facilitadoras, de onde foram extraídas as boas práticas. 
Quadro 1: Exemplo de atividades de boas práticas identificadas.

\begin{tabular}{|c|c|c|c|c|c|}
\hline Atividades (boas práticas) & $\begin{array}{l}\text { Categoria(s) de } \\
\text { perda }[11,12]\end{array}$ & $\begin{array}{l}\text { É uma atividade } \\
\text { facilitadora? }\end{array}$ & $\begin{array}{l}\text { Categoria de } \\
\text { atividade } \\
\text { facilitadora }\end{array}$ & $\begin{array}{l}\text { Nível de } \\
\text { aplicação }\end{array}$ & $\begin{array}{l}\text { Frequência nos } \\
\text { exemplos } \\
\text { pesquisados }(\%)\end{array}$ \\
\hline $\begin{array}{l}\text { Verificar qualidade do material recebido (coloração, quebras, } \\
\text { empenamentos ou em desacordo com as especificações de } \\
\text { projeto). }\end{array}$ & $\begin{array}{l}\text { Defeitos, } \\
\text { Transporte }\end{array}$ & Não & $\begin{array}{l}\text { Preparação do } \\
\text { trabalho }\end{array}$ & Operacional & 8,60 \\
\hline $\begin{array}{l}\text { Disponibilizar os equipamentos de segurança individual } \\
\text { necessários. }\end{array}$ & Espera & Sim & $\begin{array}{l}\text { Proteção dos } \\
\text { operários }\end{array}$ & Operacional & 8,60 \\
\hline $\begin{array}{l}\text { Disponibilizar peças e ferramentas de apoio antes do início do } \\
\text { serviço. }\end{array}$ & $\begin{array}{l}\text { Making-do, } \\
\text { Processamento }\end{array}$ & Não & $\begin{array}{l}\text { Preparação do } \\
\text { trabalho }\end{array}$ & Operacional & 8,60 \\
\hline $\begin{array}{l}\text { Armazenar adequadamente as peças para evitar quebras ou } \\
\text { empenas. }\end{array}$ & $\begin{array}{l}\text { Estoque, } \\
\text { Defeitos }\end{array}$ & Não & $\begin{array}{l}\text { Preparação do } \\
\text { trabalho }\end{array}$ & Operacional & 7,53 \\
\hline Observar a preparação antecipada de argamassa. & $\begin{array}{l}\text { Making-do, } \\
\text { Espera }\end{array}$ & Sim & $\begin{array}{l}\text { Preparação do } \\
\text { trabalho }\end{array}$ & Operacional & 6,45 \\
\hline $\begin{array}{l}\text { Observar os meios de transporte para levar a argamassa até o local } \\
\text { de trabalho. }\end{array}$ & $\begin{array}{l}\text { Making-do, } \\
\text { Espera }\end{array}$ & Não & Acesso & Operacional & 6,45 \\
\hline $\begin{array}{l}\text { Definir os pacotes de trabalho para evitar interrupções com perda } \\
\text { da qualidade do produto executado. }\end{array}$ & $\begin{array}{l}\text { Processamento, } \\
\text { Espera }\end{array}$ & Sim & Sequenciamento & Tático & 5,38 \\
\hline $\begin{array}{l}\text { Conferir o serviço a cada etapa de execução para evitar } \\
\text { retrabalhos por erros construtivos. }\end{array}$ & Processamento & Não & $\begin{array}{l}\text { Conferência do } \\
\text { trabalho }\end{array}$ & Operacional & 4,84 \\
\hline Determinar por onde vai iniciar o serviço. & $\begin{array}{l}\text { Making-do, } \\
\text { Espera }\end{array}$ & Não & Sequenciamento & Tático & 3,76 \\
\hline $\begin{array}{l}\text { Verificar o nivelamento da superfície antes de iniciar o serviço } \\
\text { para evitar interrupções durante o assentamento. }\end{array}$ & Espera & Sim & $\begin{array}{l}\text { Preparação do } \\
\text { trabalho }\end{array}$ & Operacional & 3,23 \\
\hline
\end{tabular}


A análise das respostas do checklist fornecidas pelos gerentes não foi realizada isoladamente, mas em conjunto com aspectos observados durante as visitas realizadas nas obras. Dessa forma, foi possível realizar uma comparação, buscando estabelecer uma relação, entre as opiniões dos gerentes e o que acontece na prática nos canteiros.

Após aplicação do checklist, todos os dados foram inseridos em uma única planilha em que as respostas dos seis gerentes foram colocadas lado a lado, para possibilitar a análise das informações.

Como resultado da aplicação do checklist, elaborou-se o gráfico da Figura 3, com base no somatório dos graus de importância atribuídos a cada uma das categorias. Dessa forma, observou-se que as quatro categorias que atingiram maiores porcentagens de atividades consideradas "importantes" ou "muito importantes" foram, em ordem decrescente, "Proteção dos Operários" (100\%), "Projeto" $(96,67 \%)$ e, com a mesma porcentagem, as categorias "Conferência do Trabalho" (91,67\%) e "Sequenciamento" (91,67\%).

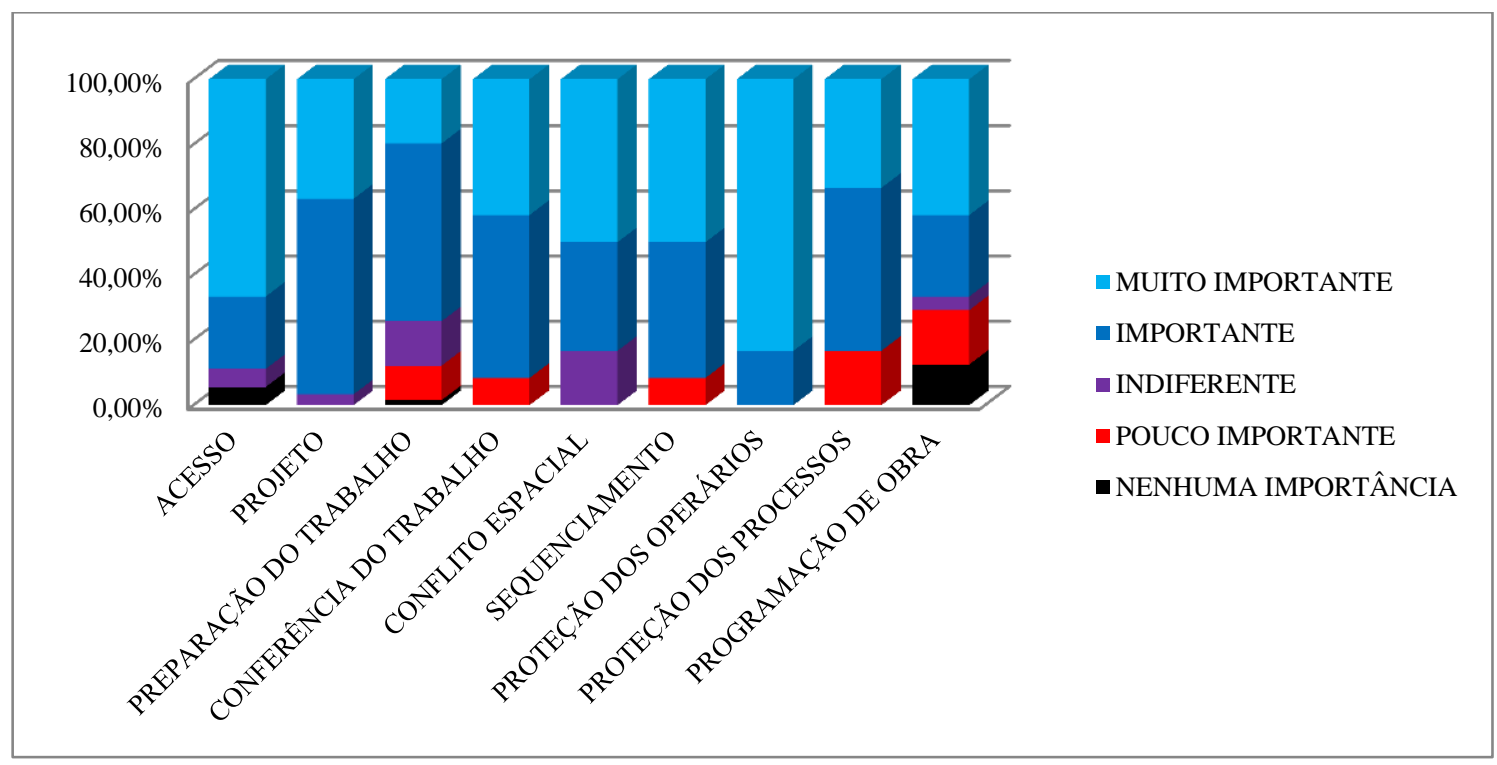

Figura 3: Respostas dos gerentes em termos de percentagem de importância por categoria de atividades.

Já o elevado grau de importância atribuído à categoria "Projeto" pode ser explicado pela necessidade em dispor de todos os projetos no local da obra, para serem consultados sempre que necessário no local de execução dos serviços, e não somente no escritório. A adoção dessa boa prática evita a ocorrência de making-do e, consequentemente, reduz a probabilidade de ocorrência de retrabalhos. Durante as visitas, constatou-se que somente duas das seis empresas pesquisadas dispunham dos projetos no local de trabalho. Logo, os gestores entendem que é importante a consulta frequente aos projetos no local de execução dos serviços, porém, nem todos tomam iniciativas no sentido de disponibilizá-los no local de trabalho e limitam sua consulta somente ao escritório da obra.

O gráfico da Figura 3 também indica a preocupação dos gerentes em somar esforços para garantir as tolerâncias dimensionais das paredes através da frequente conferência do trabalho executado. Porém, as visitas realizadas em muitas das obras indicaram falhas ocorridas na execução de paredes advindas provavelmente da falta de conferência de prumo e/ou alinhamento, gerando a necessidade de retrabalhos. Os graus de importância atribuídos pelos gerentes a partir do checklist para a conferência do trabalho podem refletir uma preocupação ou até mesmo uma necessidade existente em seus processos executivos.

Os gestores consideraram de extrema relevância a adoção de boas práticas relacionadas à categoria "Sequenciamento", o que pode evidenciar o desejo destes em manter a ordem de produção e garantir a continuidade do fluxo de produção, através de um bom planejamento, mesmo que esse não seja considerado um ponto forte dentro do seu processo executivo. Essa 
situação é facilmente perceptível ao analisar a elevada frequência das respostas "nenhuma importância", "pouco importante" ou "indiferente" fornecidas pelos gerentes para a categoria "Programação de Obra", cujo percentual foi de 33,33\%. Outra evidência de que existem falhas no planejamento é que pode haver atividades sendo iniciadas sem que todos os recursos necessários estejam disponíveis, já que se constatou um baixo grau de importância atribuído à categoria "Preparação do Trabalho" $(25,76 \%$ dos gerentes atribuíram para essa categoria as respostas "nenhuma importância", "pouco importante" ou "indiferente"). Tal fato pode culminar na ocorrência de improvisações no processo (making-do).

Observa-se que as categorias de atividades destacadas em campo, por meio da lista de boas práticas, e as evidenciadas pelos gerentes de obras são diferentes. Na lista de boas práticas, conforme já comentado, o destaque foi para as categorias "Preparação do trabalho" e "Sequenciamento", já para os gestores o destaque (assinalado como muito importante e importante) foi para as categorias "Proteção dos operários", "Projeto", "Sequenciamento" e "Conferência do trabalho". Verifica-se que as atividades pertencentes à categoria "Preparação do trabalho" são frequentes em obra e relacionam-se com a perda por making-do, em sua maioria representada por retrabalhos, ao não estarem disponibilizados os sete fluxos no momento adequado [14]. Logo, deveriam ser evidenciadas por todos os agentes envolvidos no planejamento das ações desenvolvidas em canteiro de obras.

\section{CONCLUSÃO}

A elaboração da lista de boas práticas foi fruto de pesquisa de dissertação de mestrado, que teve como principal objetivo o desenvolvimento de uma ferramenta que auxiliasse os gerentes de obras no uso das boas práticas no processo construtivo de elevação da alvenaria estrutural.

Essa etapa permitiu concluir que, de todas as boas práticas, as atividades facilitadoras merecem atenção especial dentro do processo produtivo, já que podem causar interrupções quando não são atendidas. Portanto, as empresas devem buscar formas de alertar aos gerentes quanto à importância da utilização dessas atividades no fluxo de produção.

O processo de investigação de campo, quanto à utilização das boas práticas, mostrou que os gestores, mesmo sem perceber, utilizam boas práticas no seu dia a dia e que a negligência de algumas delas pode comprometer a continuidade do processo e aumentar a probabilidade de ocorrência de making-do e, consequentemente, de retrabalhos.

\section{REFERÊNCIAS BIBLIOGRÁFICAS}

1. Santos DG, Grosskopf J, Souza AM, Santos Neto AT, Heineck LFM. Utilization of extra planning activities by construction companies in Sergipe/Brazil. In: Annual Conference of the International Group for Lean Construction, 20th, 2012, San Diego, California, United States. Proceedings... San Diego, 2012. 11p.

2. Santos DG. Modelo de gestão de processos na construção civil para identificação de atividades facilitadoras. 2004. Tese (Doutorado) - Programa de Pós-Graduação em Engenharia de Produção da Universidade Federal de Santa Catarina. Florianópolis. 2004.

3. Machado RL. A sistematização de antecipações gerenciais no planejamento da produção de sistemas da construção civil. 2003. Tese (Doutorado) - Programa de Pós-graduação em Engenharia de Produção da Universidade Federal de Santa Catarina. Florianópolis. 2003.

4. Mesquita VF. Desenvolvimento de jogo didático para tornar prático o uso das atividades que contribuem para a melhoria de processo: elevação da alvenaria estrutural. 2014. 168p. Dissertação (Engenharia Civil). Programa de Pós-Graduação em Engenharia Civil. Universidade Federal de Sergipe, São Cristóvão. 2014.

5. Bernardes MMS. Desenvolvimento de um modelo de planejamento e controle da produção para micro e pequenas empresas de construção. 2001. 291p. Tese (Doutorado em Engenharia Civil), Universidade Federal do Rio Grande do Sul, Programa de Pós-graduação em Engenharia Civil — PPGEC/UFRGS, Porto Alegre.

6. Bernardes MMS, Bortolazza R. C. Estado da arte do processo de identificação de restrições em um grupo de empresas de construção civil de Porto Alegre/RS. In: Encontro Nacional de Tecnologia do Ambiente Construído, X, 2004, São Paulo. Anais... São Paulo. 2004, 14p. 
7. Codinhoto, R. et al. Análise de restrições: definição e indicador de desempenho. In: Simpósio Brasileiro de Gestão e Economia da Construção, III, 2003, São Carlos, SP. Anais... São Carlos, 2003.

8. Treville S, Antonakis J. Could lean production job design be intrinsically motivating? Contextual, configurational, and levels-of-analysis issues. Journal of Operations Management, 2006; 24(2): 99123.

9. Liker JK, Meier DP. O talento Toyota: o modelo Toyota aplicado ao desenvolvimento de pessoas. Porto Alegre: Bookman, 2008. 296p.

10. Ohno T. O Sistema Toyota de Produção: além da produção em larga escala. Porto Alegre: Bookman, 1997. 149p.

11. Shingo S. O Sistema Toyota de Produção do ponto de vista da engenharia de produção. Porto Alegre: Artes Médicas, 1996. 291p.

12. Koskela L. Making-do - The Eight Category of waste. In: Conference of the International Group for Lean Construction, 12., 2004, Dinamarca. Proceedings... Dinamarca. 2004.

13. Leshno M, Ronen B. The complete kit concept - implementation in the health care system. Human Systems Management, 2001; 20: 313-318.

14. Koskela L. An exploration towards a production theory and its application to construction. 2000. 298p. Doctor of Philosophy, Helsinki University of Technology, VTT Technical Research Centre of Finland, Espoo. 2000.

15. Sommer L. Contribuições para um método de identificação de perdas por improvisação em canteiros de obras. 2010. Dissertação (Mestrado em Engenharia Civil). Programa de Pós-Graduação em Engenharia Civil, Universidade Federal do Rio Grande do Sul, Porto Alegre. 2010.

16. Fireman MCT. Proposta de método de controle integrado produção e qualidade, com ênfase na medição de perdas por making-do e retrabalho. 2012. Dissertação (Mestrado) - Programa de PósGraduação em Engenharia Civil da Universidade Federal do Rio Grande do Sul. Porto Alegre. 2012.

17. Coelho CB. T. Antecipações gerenciais para a inserção de atividades facilitadoras na tarefa de execução de alvenaria de tijolos cerâmicos: relato de agentes do processo. 2009. Dissertação (Mestrado em Construção Civil). Programa de Pós-Graduação em Construção Civil. Universidade Federal do Paraná. Curitiba. 2009.

18. Formoso CT, Sommer L, Koskela L, Isatto EL. An exploratory study on the measurement and analysis of making-do in construction sites. In: Conference of the International Group for Lean Construction, 19., 2011, Lima. Proceedings... Lima. 2011.

19. Fireman, M. C. T.; Formoso, C. T. Integrating Production and Quality Control: Monitoring MakingDo and Unfinished Work. In: Annual Conference of the International Group for Lean Construction, 21th, 2013, Fortaleza, Brazil. Proceedings... Fortaleza, 2013. p 515-525.

20. Grosskopf J. Boas práticas e descontinuidades: do projeto à execução de um empreendimento complexo. 2013. 75p. Dissertação (Mestre em Engenharia Civil). Programa de Pós-Graduação em Engenharia Civil. Universidade Federal de Sergipe, São Cristóvão. 2013.

21. Leão CF. Proposta de modelo para controle integrado da produção e da qualidade utilizando tecnologia de informação. 2014. 179p. Dissertação (Mestrado em Engenharia) - Programa de PósGraduação em Engenharia Civil, Universidade Federal do Rio Grande do Sul, Porto Alegre. 2014.

22. Formoso CT, Isatto EL, Fireman MC. T. Integrating production and quality control: monitoring making-do and unfinished work. In: Conference of the International Group for Lean Construction, 21., 2013, Fortaleza. Proceedings... Fortaleza, 2013.

23. Koskela L. Application of the new production philosophy to construction. Technical Report $n^{\circ}$. 72. Center of Integrated Facility Engineering. Department of Civil Engineering. Standford University, 1992, $72 \mathrm{p}$. 